\title{
Manejo de pesquerías mediante la zonificación de áreas de reserva: aplicación a la pesquería de la anchoveta (Engraulis ringens) en el norte de Chile*
}

\author{
Juan Pablo Díaz ${ }^{1}$ \& Hugo Salgado ${ }^{2}$ \\ ${ }^{1}$ Universidad Arturo Prat, Casilla 121, Iquique, Chile \\ ${ }^{2}$ Departamento de Economía, Universidad de Concepción, Casilla 1987, Concepción, Chile
}

\begin{abstract}
RESUMEN. Se presentan las bases metodológicas para resolver la problemática surgida al establecer ventanas de penetración en el Area de Reserva a la Pesca Artesanal (ARPA) mediante un modelo bioeconómico en la pesquería de anchoveta (Engraulis ringens) en la región norte de Chile. Se evalúa y analiza la pesquería en dos escenarios de política de administración. El ARPA es incorporada como una variable de decisión en la administración del recurso, donde también se establece su relación con otras medidas de manejo, como esfuerzo de pesca y cuota global. Para dar sustento metodológico al modelamiento, se han tomado conceptos de la teoría sobre reservas marinas, los cuales han sido adaptados a una situación homóloga con el ARPA. Los resultados permiten afirmar que es posible manejar la pesquería de la anchoveta en la zona norte, mediante la determinación del porcentaje del ARPA que se otorga al sector industrial vía ventana de penetración.
\end{abstract}

Palabras clave: anchoveta, pesquería pelágica, regulación pesquera, pesca artesanal, pesca industrial, Chile.

\section{Fishery management through the zoning of reserve areas in the anchovy (Engraulis ringens) fishery off northern Chile*}

\begin{abstract}
The methodological bases are presented for solving the problem that arises upon establishing "windows of penetration" in an Area Reserved for Small-scale Fishing (ARPA) through a bioeconomic model of the anchovy (Engraulis ringens) off northern Chile. The fishery is analyzed and evaluated given two scenarios of administrative policy. The ARPA is incorporated as a decision variable in the resource administration, and its relationships with other means of management (e.g., fishing effort and global quotas) are also established. The model is supported methodologically, taking concepts from the theory of marine reserves and adapting these to a homologous situation with the ARPA. The results show that it is possible to manage the anchovy fishery in the northern zone by determining the percentage of the ARPA that is granted to the industrial sector through the windows of penetration.
\end{abstract}

Key words: anchovy, pelagic fishery, fishery regulation, small-scale fishing, industrial fishing, Chile.

Autor corresponsal: Juan P. Díaz (jpdiaz@unap.cl)

\section{INTRODUCCIÓN}

El sector pesquero industrial que opera en la región norte de Chile fue pionero en el desarrollo de la pesca pelágica en el país. En efecto, fue a mediados de los años 50 cuando se inició en Arica esta actividad con el establecimiento de una planta reductora, para luego a fines de esa década y comienzos de los 60, se expandió bajo el amparo de políticas oficiales de incentivos tributarios a los puertos de Iquique, Tocopilla y Mejillones. Sucesivas crisis de baja disponibilidad en los recursos pesqueros vividas en el transcurso de los años, en su mayor parte asociadas a la ocurrencia de los eventos de El Niño, han ido modificando la estructura industrial pesquera, la que a pesar de los vaivenes propios de esta actividad se

* Trabajo presentado en el XXV Congreso de Ciencias del Mar de Chile y XI Congreso Latinoamericano de Ciencias del Mar (COLACMAR), realizados en Viña del Mar, entre el 16 y 20 de mayo de 2005. 
ha logrado mantener en el tiempo, dando trabajo y bienestar a parte importante de la población en la zona norte. En la actualidad, la actividad de la industria pesquera en el norte de Chile está fuertemente asociada a la disponibilidad del recurso anchoveta (Peña-Torres et al., 2003).

La Ley General de Pesca y Acuicultura, en el artículo 47, ha reservado para el ejercicio de actividades pesqueras extractivas artesanales una franja de mar territorial de cinco millas, la cual es denominada Área de Reserva a la Pesca Artesanal (ARPA). Sin embargo, la Ley señala de modo específico que cuando en esta zona de reserva no se realicen actividades pesqueras extractivas artesanales o si las hubiere, sea posible el desarrollo de actividades pesqueras extractivas por la flota industrial, se podrá autorizar de modo transitorio la operación de esta última, previo informe técnico del Consejo Zonal de Pesca. Así, entonces, periódicamente la autoridad ha debido revisar sobre la base de los antecedentes técnicos el estado de interacción entre ambos sectores, para luego emitir una resolución señalando las áreas o "ventanas de penetración" de la flota industrial en el área de reserva a la pesca artesanal y el periodo en la cual se aplica esta excepción.

La contribución del presente trabajo es demostrar que el tamaño del ARPA puede ser considerada como una nueva y eficiente medida de manejo en la pesquería de anchoveta, considerando el intercambio entre el sector industrial y artesanal. Esto se evalúa mediante un modelo bioeconómico y se indican políticas de administración de la pesquería, ante las solicitudes de ventanas de penetración de la flota industrial en el ARPA. Además, ésta área es incorporada como variable de decisión en la administración del recurso, donde se considera su relación con el establecimiento de una cuota global. En tal sentido, para dar un sustento metodológico al modelamiento, es que se han tomado conceptos de la teoría existente sobre reservas marinas, los cuales han sido adaptados a una situación homóloga con el área de reserva a la pesca artesanal.

\section{MATERIALES Y MÉTODOS}

\section{Modelamiento bioeconómico-estático}

El modelo bioeconómico desarrollado en esta investigación considera aspectos teóricos tomados de Boncoeur et al. (2002) y Sanchirico \& Wilen (2001). El propósito de los primeros, es presentar un modelo de simulación que considera las consecuencias económicas de llevar a cabo una reserva marina en un sistema de predador-presa. El modelo permite simular los impactos de varias políticas, en lo que se refiere al tamaño de la reserva, sobre las rentas económicas generadas por ambas pesquerías comerciales y la industria del ecoturismo. El análisis se realiza con un modelo bioeconómico que combina los rasgos del modelo de la reserva marina y el modelo de multi-especies. La dinámica de los stock es modelada por dichos autores como sigue:

$$
\begin{aligned}
& \frac{d X_{1}}{d t}=\mathrm{r}_{\mathrm{F}} \cdot \mathrm{X}_{\mathrm{F} 1} \cdot\left(1-\frac{\mathrm{X}_{\mathrm{F} 1}}{\alpha \cdot X_{F \max }}\right)-T-\beta \cdot X_{F 1} \cdot X_{M} \\
& \frac{d X_{2}}{d t}=\mathrm{r}_{\mathrm{F}} \cdot \mathrm{X}_{\mathrm{F} 2} \cdot\left(1-\frac{\mathrm{X}_{\mathrm{F} 2}}{(1-\alpha) \cdot X_{F \max }}\right)+T-\beta \cdot X_{F 2} \cdot X_{M}-Y_{F 2} \\
& \frac{d X_{M}}{d t}=\mathrm{r}_{\mathrm{M}} \cdot \mathrm{X}_{\mathrm{M}} \cdot\left(1-\frac{\gamma \mathrm{X}_{\mathrm{M}}}{X_{F 1}+X_{F 2}}\right)
\end{aligned}
$$

donde:

$X_{F i}$ : fracción de la biomasa del stock de peces en la sub-región i, el i = 1, 2 (donde 1 es la reserva marina y 2 es la zona de pesca).

$X_{M}$ : biomasa del stock de focas.

$r_{F} \quad$ : tasa intrínseca de crecimiento de la biomasa del stock de peces.

$X_{F \max }$ : máxima biomasa del stock de peces del total de la región bajo estudio.

$T$ : transferencia neta de biomasa de la reserva marina a la región de pesca.

$\beta \quad$ : tasa instantánea de mortalidad por pesca producto de la predación por focas.

$\gamma \quad$ : tasa de equilibrio de biomasa del pez-afoca.

$Y_{F 2}$ : captura de peces en la región abierta a la pesca.

$\alpha \quad$ : nivel de biomasa protegida.

En este caso, se ha tomado como base el modelo anteriormente presentado. Donde se ha eliminado la componente del ecoturismo (focas) y se ha incorporado la pesca artesanal en el área de reserva y se ha dejado la pesca industrial en el área no protegida. De esta manera se representa la problemática del ARPA.

El modelo bioeconómico propuesto considera una población donde hay dos zonas discretas en el espacio, cada una de las cuales se caracteriza por su propia dinámica y la unión entre "parches". Se 
supone que el área bajo estudio será dividida en dos sub-espacios (parches): Uno de reserva (ARPA), es decir, un espacio donde se prohibe la pesca industrial pero se permite la artesanal (espacio 1) y otro abierto a la flota industrial, es decir, ventana de penetración (espacio 2).

La dinámica del stock en cada zona es como sigue:

$$
\begin{aligned}
& \frac{d X_{1}}{d t}=\gamma_{\mathrm{F}} * X_{1} *\left(1-\frac{\mathrm{X}_{1}}{\alpha * X_{\max }}\right)-T-q_{A} * \bar{E}_{A} * X_{1} \\
& \frac{d X_{2}}{d t}=\gamma_{\mathrm{F}} * \mathrm{X}_{2} *\left(1-\frac{\mathrm{X}_{2}}{(1-\alpha) * X_{\max }}\right)+T-q_{I} * E_{I} * X_{2} \\
& T=b *\left(\frac{X_{1}}{X_{\max }}\right)
\end{aligned}
$$

Para mejor comprensión sea:

$\mathrm{X}_{\mathrm{T}}: \mathrm{X}_{1}+\mathrm{X}_{2}$ (biomasa total presente en el ARPA),

$\mathrm{X}_{1}$ : fracción de biomasa presente en el área protegida, $\mathrm{y} \mathrm{X}_{1}=\alpha^{*} \mathrm{X}_{\mathrm{T}} \mathrm{y} \mathrm{X}_{2}=(1-\alpha)^{*} \mathrm{X}_{\mathrm{T}}$

donde:

$\mathrm{X}_{\mathrm{T}}$ : biomasa total.

$\mathrm{X}_{\mathrm{i}}$ : biomasa del stock de peces en la sub-región $\mathrm{i}$, donde $\mathrm{i}=1,2$ ( 1 es la reserva a la pesca artesanal y 2 es la zona de pesca industrial).

$\gamma_{\mathrm{F}}$ : tasa intrínseca de crecimiento de la biomasa del stock de peces (Se supone igual en ambos sectores, dada la existencia de las mismas condiciones ambientales).

$\mathrm{X}_{\text {máx }}$ : capacidad de carga de peces en la región total bajo estudio.

$\mathrm{T}$ : transferencia neta de biomasa de la reserva artesanal a la región de pesca industrial.

$\mathrm{q}_{\mathrm{j}} \quad$ : coeficiente de capturabilidad, donde: $\mathrm{j}=\mathrm{A}=$ artesanal y $\mathrm{j}=\mathrm{I}=$ industrial.

$E_{j} \quad$ : esfuerzo de pesca, donde: $j=A=$ artesanal y $\mathrm{j}=\mathrm{I}=$ industrial.

$\alpha \quad$ : porción de reserva a la pesca artesanal o porcentaje de protección.

Los parches son caracterizados por las ecuaciones de movimiento del stock 4 y 5 , que tienen incorporado el proceso del crecimiento biológico mediante el modelo logístico clásico.

Para completar el modelo, sólo falta incorporar un mecanismo de transferencia de biomasa (T). Dada la naturaleza del problema, en especial la carencia de literatura sobre este tema, es que se han considerado los conceptos presentados por Sanchirico \& Wilen
(2001), sólo en lo que respecta al mecanismo de transferencia. Estos señalan que una exploración seria del diseño de reserva debe incorporar conceptos ecológicos importantes que traten el rol del espacio de los sistemas biológicos y la manera que el espacio afecta los procesos fundamentales. Dada la importancia particular a las nociones de recurso "parches" (patch) y heterogeneidad, uniones biofísicas y mecanismos de dispersión de uniones de parches. Estos conceptos se están usando para administrar reservas terrestres y resolver problemas como: corredores, bordes y configuraciones del parche que afectan la viabilidad de la especie y la diversidad, y cómo se mantiene el tamaño de la población viable mediante la dispersión espacial.

En el proceso de transferencia de biomasa desde la reserva hacia el área de pesca, Sanchirico \& Wilen (2001) consideran tres mecanismos:

a) Sistema cerrado: es decir, no existe dispersión de biomasa entre las áreas, donde no hay ningún cambio en la parte abierta a la pesca del sistema como resultado de la reserva. Así, las reservas de este tipo contribuyen a la conservación pública y a los objetivos de biodiversidad, pero a expensas de la industria;

b) Sistema "Sink-Source": las biomasas fluyen desde un parche a un segundo parche con flujo unidireccional, generalmente asumidos como resultado de procesos oceanográficos como corrientes, vientos y temperatura, que dicen relación con el ciclo de vida de la especie (migraciones por desove o reclutamiento); $y$

c) Sistema de densidad dependiente: en este caso existe un parámetro de dispersión y la biomasa fluye de un parche a otro, dependiente de las densidades relativas de los parches a las capacidades de carga natural. Eso es equivalente a asumir que existe migración de peces, desde los parches con mayor densidad hacia los de menor densidad, hasta que las densidades se igualan.

Sanchirico \& Wilen (2001) mediante un modelo de explotación espacial encuentran los equilibrios de esfuerzo y pesca en la situación de pre-reserva y reserva. El modelo usado considera a pescadores que operan bajo las condiciones de acceso abierto, respondiendo a las ganancias, entrando hasta que las rentas netas son cero, como lo plantean Gordon (1954) y Smith (1968). A estos elementos, Sanchirico \& Wilen (2001) le incorporan un componente de distribución espacial a la dispersión biológica de la población. 
Igualmente, Sanchirico \& Wilen (2001) manifiestan que con el establecimiento de un sistema de reserva se cerrarán áreas (parches) sobreexplotadas, donde el efecto inicial en la reserva es el crecimiento de la biomasa. Como la biomasa en las áreas cerradas crece, se generarán diferenciales de densidad entre áreas cerradas y áreas abiertas (pesca), causando nuevos modelos de dispersión de la biomasa en las áreas abiertas. Pero la dispersión de biomasa a su vez genera nuevos modelos de rentas relativas sobre el espacio, llevando a una reordenación del esfuerzo. A la larga, una nueva distribución de equilibrio de biomasa, esfuerzo y captura surgirá, y se podría comparar directamente pre-reserva y equilibrio de post-reserva. Afortunadamente, un sistema de dos parches es suficiente para caracterizar las implicaciones cualitativas de formación de la reserva bajo la mayoría de los escenarios. Se considera que ambos parches pueden ser caracterizados por el proceso logístico propio del crecimiento biológico, $f\left(x_{i}\right)=$ $r_{F i}\left(1-x_{i} / X F_{\text {max }_{i}}\right)$ con tasas de crecimiento intrínsecas $r_{F i}$ y capacidad de carga $X_{F \max i}(\operatorname{con} i=1,2)$. Ahora normalizado y definido $X_{i} \equiv\left(x_{i} / X_{F \max i}\right)$ para que en lugar de evaluar la abundancia de la biomasa, se evalúe la densidad de la biomasa en cada parche.

En este caso, solamente se ilustrarán los estados bioeconómicos para el caso de pre-reserva, el cual se puede escribir formalmente como:

$$
\begin{aligned}
& \dot{x}=0 \Rightarrow[F(x)+D] x-H(E, x)=0 \\
& \dot{E}=0 \Rightarrow S R(E, x)+\Delta R(E, x)=(S+\Delta) R(E, x)=0
\end{aligned}
$$

donde: $\mathrm{F}(\mathrm{x})$ (matriz del proceso logístico del crecimiento biológico), D (matriz de dispersión biológica), x (biomasa), H (E,x) (vector de proporciones de cosecha dependientes de biomasa y esfuerzo), SR $(\mathrm{E}, \mathrm{x})$ (captura los cambios del esfuerzo respondiendo a los niveles de renta), $\Delta \mathrm{R}(\mathrm{E}, \mathrm{x})$ (dispersión espacial de rentas. Habrá dispersión entre parche i-j, si las rentas se exceden entre ellas.
Mientras la matriz de coeficientes de dispersión biológica afecta al vector de equilibrio de biomasa y el nivel de esfuerzo en cada parche, la matriz de parámetros de la respuesta económica sólo afecta la velocidad de respuesta al equilibrio. Esto ocurre porque el sistema económico se equilibra cuando las rentas netas en cada parche son cero y las condiciones que generan rentas cero son independientes del parámetro de captura.

Los autores asumen que la función de producción es una función de Schaefer para que la cosecha sea $h_{i}=q_{i}{ }^{*} E_{i} * X_{i}$ y la función del costo es lineal en el esfuerzo, tal que $C\left(E_{j}\right)=c_{i} * E_{i}$. Se debe notar que con estas especificaciones, las ecuaciones de la renta son separables, de norma que: $R(E i X i)=R(X i) * E i$, donde $R\left(X_{i}\right)=p_{i} * q_{i} * X_{i}-\mathrm{c}_{\mathrm{i}}$. Con la separabilidad, las rentas son disipadas en cada parche cuando el nivel de equilibrio de densidades de biomasa es $\mathrm{X}_{\mathrm{i}}^{\mathrm{ss}}=w_{i} \equiv\left[\left(\mathrm{c}_{\mathrm{i}}\right) / p_{i}^{*} q_{i}\right]$. Las densidades de biomasa de equilibrio son funciones de la función de producción y parámetros de función de costo. Una vez que el $\mathrm{X}_{i}^{\mathrm{ss}}$ es determinado, ellos pueden ser reemplazados en las ecuaciones respectivas (7) describiendo el equilibrio biológico en cada parche para determinar el correspondiente nivel de esfuerzo de equilibrio (Tabla 1).

De acuerdo a Yáñez et al. (1995) las distribuciones espaciales y mensuales de la anchoveta presentan variaciones que se asocian a las modificaciones de la estructura térmica superficial del mar. Este cambio de distribuciones espaciales puede ser explicado por el mecanismo de "Sink-Source", el cual posibilitaría la estimación de una transferencia de biomasa " $b$ " propio de la especie.

La ecuación 6 permite representar la transferencia neta de biomasa de peces (T) desde el ARPA al área de pesca industrial. Donde ésta es proporcional al tamaño relativo de la biomasa de peces hasta llegar a la capacidad de carga. Para la estimación del parámetro b de la ecuación 6 , se utilizarán las ecuaciones de equilibrio bioeconómico en pre-reserva descritas por Sanchirico \& Wilen (2001). El modelo usado

Tabla 1. Equilibrio bio-económico en pre-reserva (Fuente: Sanchirico \& Wilen, 2001).

Table 1. Bio-economic balance in pre-reservation (Source: Sanchirico \& Wilen, 2001).

\begin{tabular}{cccc}
\hline $\begin{array}{c}\text { Sistema de } \\
\text { dispersión }\end{array}$ & $\begin{array}{c}\text { Densidad de } \\
\text { biomasa }\end{array}$ & Niveles de esfuerzo & Niveles de cosechas (Captura) \\
\hline Sink-source & $\mathrm{w}_{\mathrm{i}}$ & $E_{i}=\frac{r_{F i}}{q_{i}}\left(1-w_{i}\right)-\frac{b}{q_{i}}$ & $H_{i}=r_{F i} w_{i}\left(1-w_{i}\right)-b w_{i}$ \\
\hline
\end{tabular}


considera a pescadores que operan bajo las condiciones de acceso abierto. Por este motivo, se usarán los datos de la pesquería entre 1986 y 1993, ya que en ese periodo existió acceso abierto a la pesca de la anchoveta en el ARPA. Es decir, la pesquería estaba en condición de pre-reserva, puesto que desde 1994 en adelante se restringe el acceso a la flota industrial al ARPA. También estos autores plantean ecuaciones para estimar el parámetro b en la condición de reserva, bajo el supuesto de libre acceso a la pesquería, situación que en esta pesquería no se cumple en el periodo 1994-2002. La condición de pre-reserva es necesaria para poder aplicar las ecuaciones 8 y 9 propuestas por Sanchirico \& Wilen (2001).

$$
\begin{aligned}
& E_{j}=\frac{\gamma_{F}}{q} *\left(1-w_{j}\right)-\frac{b}{q} \\
& Y_{j}=\gamma_{F} * w_{j}\left(1-w_{j}\right)-b * w_{j}
\end{aligned}
$$

donde:

$\mathrm{E}_{\mathrm{j}} \quad$ : esfuerzo año $\mathrm{j}$.

$\mathrm{w}_{\mathrm{j}}$ : densidad de biomasa $\left(\mathrm{X}_{1} / \mathrm{X}_{\max }\right)$ año $\mathrm{j}$. Supuesto: $X_{1} \neq X_{\max }$, dado que por existir pesca las biomasas no alcanzarán los valores de la capacidad de carga.

$\mathrm{Y}_{\mathrm{j}} \quad$ : captura año $\mathrm{j}$.

B : transferencia de biomasa.

q : coeficiente de capturabilidad de la pesquería.

Resolviendo el sistema de ecuaciones 8 y 9, se obtienen los valores de $w$ y $b$

$$
\begin{aligned}
& w_{j}=\frac{Y_{j}}{E_{j} * q} \quad \begin{array}{l}
\text { remplazando en } 7, \\
\text { es factible despejar b }
\end{array} \\
& b=\gamma_{F}\left(1-\frac{Y_{j}}{E_{j} * q}\right)-E_{j}^{*} q
\end{aligned}
$$

Si se considera que los cambios en biomasa se representan de acuerdo a las ecuaciones 4 y 5 , donde la condición de equilibrio se establece cuando $\left(d X_{i}\right)$ $d t)=0$, entonces es factible despejar la biomasa de la población en equilibrio, para ambos casos $X e_{1} \mathrm{y}$ $\mathrm{Xe}_{2}$.

Con las biomasas en equilibrio es posible estimar los excedentes productivos $(G(x))$, en función del esfuerzo de pesca industrial y los niveles de protección, garantizando la pesca artesanal.

$$
G(x)=\gamma_{F} * X e_{2} *\left(1-\frac{X e_{2}}{X_{\max }}\right)+\gamma_{F} * X e_{1} *\left(1-\frac{X e_{1}}{X_{\max }}\right)
$$

Se supone que la captura por unidad de esfuerzo es proporcional a la densidad de peces. En este estudio la actividad de pesca artesanal se considerará sin crecimiento (Peña-Torres et al., 2003; Díaz et al., 1997). Dada la dificultad de estimar un precio para la captura, se considerará el procedimiento propuesto por Salgado (2000), ya que no existe información que refleje el verdadero precio del recurso anchoveta para la flota. Se considera que el verdadero valor estará dado por el beneficio neto de procesamiento que se puede obtener en la industria reductora, puesto que todas las capturas son destinadas a la producción de harina. Por otra parte, para la estimación de la captura artesanal, se considera que es del tipo Schaefer (1954). Por tanto, el modelo establece que el beneficio neto derivado de la actividad pesquera está en función de los ingresos totales sostenibles y de los costos totales. En relación a los costos del sector artesanal, se ha considerado el valor de su captura, puesto que se está estimando el beneficio de la producción de harina y no de la extracción:

$$
\pi=\left(P_{H}-C_{P}\right) * \eta * Y-C_{E} * E_{I}-C_{A} * q_{A} * \bar{E}_{A} * X e_{1}
$$

donde:

$\pi \quad$ : beneficio de la producción de harina en la pesquería.

$P_{H} \quad$ : precio por tonelada de harina.

$\mathrm{C}_{\mathrm{P}}$ : costo asociado al proceso de elaboración, es decir, costo por ton de harina producida.

Y : captura total.

$\eta \quad$ : factor de eficiencia en la producción de harina (ton de harina/ton captura, valor aproximado $21 \%)$.

$\mathrm{C}_{\mathrm{E}} \quad$ : costo por la unidad de esfuerzo aplicada.

$\mathrm{E}_{\mathrm{I}} \quad$ : esfuerzo industrial (viajes con pesca y sin pesca).

$\mathrm{C}_{\mathrm{A}}$ : costo por ton de captura obtenida por la flota artesanal.

$\mathrm{q}_{\mathrm{A}} \quad$ : coeficiente de capturabilidad artesanal.

$\mathrm{E}_{\mathrm{A}}$ : esfuerzo de pesca artesanal.

$\mathrm{Xe}_{1}$ : biomasa en equilibrio en el ARPA.

\section{Modelamiento bioeconómico dinámico}

El modelamiento bioeconómico dinámico del stock en ambas áreas, será representado por las ecuaciones diferenciales 4 y 5 del modelamiento estático, sujeto a los cambios del esfuerzo en la medida que se produ- 
cen variaciones en el beneficio. Lo anterior permitirá evaluar la pesquería bajo dos sistemas de regulación de Acceso Abierto $(A A, j=1)$. Se entenderá por AA la capacidad de la industria pesquera de aumentar o disminuir su propio esfuerzo existente, es decir, no existe la incorporación de nuevas naves y Captura Total Permisible (CTP, $\mathrm{j}=2)$, donde:

$$
\begin{aligned}
& X_{t+1}^{1}=X_{t}^{1}+\gamma_{F}^{*} X_{1}^{*}\left(1-\frac{X_{t}^{1}}{\alpha^{*} X_{\max }}\right)-b * \frac{X_{t}^{1}}{X_{\max }}-q_{A} * \bar{E}_{A} * X_{t}^{1} \\
& X_{t+1}^{2}=X_{t}^{2}+\gamma_{F}^{*} X_{T} *\left(1 \frac{X_{t}^{2}}{(1-\alpha)^{*} X_{\max }}\right)+b * \frac{X_{t}^{1}}{X_{\max }}-q_{I} * E_{I} * X_{t}^{2}
\end{aligned}
$$

Con las ecuaciones 14 y 15, los tamaños del stock en el próximo periodo se relacionan con la cantidad de biomasa existente en el periodo actual $\left(X^{1}{ }_{t} y X^{2}{ }_{t}\right)$, seguido de su crecimiento biológico y negativamente con las capturas artesanal e industrial, respectivamente.

Se supone que las empresas toman decisiones respecto a la operación de la flota conforme a los resultados obtenidos en el beneficio en el periodo anterior, sujeto a una regulación vigente. Lo anterior en este caso se reflejará en cambios en el esfuerzo y las ecuaciones que simulan este comportamiento se describen como sigue:

$$
\begin{array}{llll}
\Delta E_{j t}=\lambda \prod_{t j} & \text { si } \prod_{t j}<0 & \forall t, j \\
\Delta E_{j t}=\lambda \prod_{t j} & \text { si } \prod_{t j}>0 & \mathrm{y} \prod_{t, j}=\operatorname{Max}\left(\prod_{t, j}\right) \quad \forall t, j \\
\Delta E_{j t}=0 & \text { si } \prod_{t j}>0 & & \mathrm{y} \prod_{\mathrm{t}, \mathrm{j}}<\operatorname{Max}\left(\prod_{\mathrm{t}, \mathrm{j}}\right) \quad \forall t, j
\end{array}
$$

donde:

$\Delta \mathrm{E}_{\mathrm{jt}}$ : es la variación del esfuerzo, en el periodo $\mathrm{t}$, bajo una regulación j de la pesquería.

$\lambda \quad$ : parámetro que muestra la tasa de disminución o aumento del esfuerzo según los beneficios o pérdidas obtenidos en cada periodo (Smith, 1968).

\section{Acceso Abierto (AA)}

Bajo esta regulación las empresas no tienen restricciones sobre las capturas, hasta su máximo esfuerzo permitido, por lo tanto las capturas son del tipo Schaefer, incorporada en las ecuaciones 14 y 15. La pesquería alcanzará el equilibrio de largo plazo cuando se cumpla la condición de beneficio igual a cero. El beneficio se determina según la ecuación 13 .

\section{Captura Total Permisible (CTP)}

La captura total permisible se estima como una proporción de la biomasa (Anderson, 2000), donde la captura deberá ser sustituida por:

$$
\mathrm{CTP}_{\mathrm{t}}=\phi * \mathrm{X}_{\mathrm{t}}^{2}
$$

donde: $0<\phi<1$, parámetro positivo que representa el porcentaje de biomasa que corresponde a la captura total permisible.

\section{Cálculo de los parámetros en el modelo bioeconómico}

Caso dinámico: dada la falta de información sobre la estimación de este parámetro $\lambda$ y dada la naturaleza de esta investigación, que no busca predecir sino más bien visualizar la tendencia del ajuste del esfuerzo industrial, se ha sensibilizado el parámetro en el rango $(0,00008-0,00016)$ y analizado sus efectos en la biomasa y los beneficios de la pesquería. En relación a la biomasa a distintos niveles de protección, considerando AA y CTP, se puede afirmar que el parámetro $\lambda$, no afecta el comportamiento global de las tendencias de las biomasas, la diferencia está en los tiempos en que convergen. De la misma forma que en el caso anterior, variaciones del parámetro $\lambda$ afectan la convergencia de los beneficios en ambas situaciones (AA y CTP) y prácticamente se mantiene la tendencia en los distintos niveles de protección, pero existen pequeñas variaciones en los valores de los beneficios para la política de CTP, pero la tendencia se mantiene. En este sentido se ha considerado $\lambda=0,00012$, que en realidad corresponde a un valor que permite una convergencia no rápida de las variables en estudio.

En relación al parámetro $\phi$, se ha sensibilizado el parámetro en el rango $(0,8-0,14)$, y considerando sus efectos en la biomasa y los beneficios de la pesquería. Dichos valores pueden representar las estimaciones de cuota aplicada en la misma. Al igual que en el caso anterior, $\phi$ no afecta el comportamiento global de las tendencias de las biomasas y beneficio de la pesquería, la diferencia está en los tiempos en que convergen y se toma el valor referencial de $\phi=0,11$. Con respectos a los otros parámetros estos tomarán los mismos valores que para el modelamiento estático.

Caso Estático: las metodologías tradicionales para la estimación de la capacidad de carga y la tasa intrínseca de crecimiento, se han basado en ajustes regresionales entre la biomasa y la captura, permitiendo de esta forma obtener simultáneamente ambos parámetros. Al respecto, existe una estimación de la capacidad de carga y la tasa intrínseca de crecimiento de la pesquería de la anchoveta o en conjunto con 
la sardina, tal es el caso Böhm \& Barbieri (1996) y Aliaga et al. (2001), respectivamente. Estos resultados se caracterizan por una subestimación de la capacidad de carga y una sobreestimación de la tasa intrínseca de crecimiento. No obstante se tomarán dichos valores en forma referencial. En este caso, se estimarán ambos parámetros en forma separada. La estimación de la capacidad de carga se basa en el siguiente hecho: generalmente en el crecimiento de una población, hay periodos de tiempo en los cuales las poblaciones pueden crecer exponencialmente. Esta situación se presenta en la pesquería de la anchoveta en el periodo 1984-1993. Entonces, es posible estimar una tasa de crecimiento en esta fase, mediante el despeje de la siguiente ecuación:

$$
X_{0(1984)}=X_{0(1993)} e^{\gamma_{F}^{* 9}}
$$

El modelo exponencial indica que no importa qué tamaño tenga la población, ya que sigue creciendo a la misma tasa por individuo, lo que no es real, ya que existe un freno debido a la capacidad de carga, por tanto es necesario imponer esta condición a la ecuación, entonces:

$$
\frac{d X_{0}}{d t}=\gamma_{F} *\left(X_{\max }-X_{F 0}\right)
$$

donde:

$X_{\max }$ : capacidad de carga de peces en la región total bajo estudio.

$X_{0} \quad$ : biomasa total de peces.

De acuerdo a esto, la población crecerá a una tasa per-capita que disminuye a medida que $\mathrm{X}_{0}$ se acerca a $\mathrm{X}_{\max } \mathrm{y}$ cuando $\mathrm{X}_{0}$ es igual a $\mathrm{X}_{\max }$ los individuos aportan un balance neto igual a cero de descendencia y la población se estabiliza en el tamaño $\mathrm{X}_{0}=\mathrm{X}_{\max }$ (Claramunt et al., 2003). Para resolver el modelo de la ecuación (19), se integra y se tiene:

$$
X_{0}(t)=X_{\max }-c e-\gamma_{F}^{* t}
$$

Entonces es factible capturar el valor de $\mathrm{X}_{\max }$ mediante un ajuste regresional, cuando $t \rightarrow \infty$.

Con el valor estimado de la capacidad de carga de la pesquería $\hat{X}_{\max }$, es posible estimar la tasa de crecimiento intrínseco de la pesquería mediante el modelo logístico, donde en condiciones de equilibrio $\mathrm{dX} / \mathrm{dt}=0$, luego:

$$
\gamma_{\mathrm{F}} * \mathrm{X}_{0} *\left(1-\frac{\mathrm{X}_{0}}{\hat{X}_{\max }}\right)=q * E * X_{0}=0
$$

despejando $\gamma_{\mathrm{F}}$, se tiene:

$$
\gamma_{F}=\frac{q^{*} E^{*} \hat{X}_{\max }}{\left(\hat{X}_{\max }-X_{0}\right)}
$$

\section{Estimación del coeficiente de capturabilidad industrial}

En forma referencial se considera un valor del coeficiente de capturabilidad (q), determinado por Agüero \& González (1996) para la pesquería conjunta de anchoveta y sardina. Además, se dispone de información de biomasa, captura y captura por unidad de esfuerzo, desde 1986 hasta 2001 (IFOP, 2003).

donde:

$$
q_{j}=\left(\frac{Y_{j}}{E_{j} * X_{j}}\right)
$$

$\mathrm{X}_{\mathrm{j}} \quad$ : biomasa año $\mathrm{j}$.

$\mathrm{Y}_{\mathrm{j}} \quad$ : captura año $\mathrm{j}$.

$\mathrm{E}_{\mathrm{j}} \quad$ : esfuerzo de pesca industrial año $\mathrm{j}$.

Para la estimación del coeficiente de capturabilidad artesanal, se consideró el siguiente hecho: La CPUE (captura por unidad de esfuerzo) es lineal a la capacidad de bodega en el recurso anchoveta, con un ajuste de $r^{2}=0,87(n=34)$ (Böhm \& Barbieri, 1996). Esto indica que el coeficiente de capturabilidad cambia en la misma proporción con que cambia la CPUE. En este caso tomando la embarcación media artesanal de $80 \mathrm{~m}^{3}$ de capacidad de bodega y la industrial de $350 \mathrm{~m}^{3}$. Se esperaría que disminuya en 4,375 veces el coeficiente de capturabilidad. La obtención del coeficiente de capturabilidad, permite conocer el esfuerzo artesanal, mediante el despeje de la ecuación de captura (22).

Simulación estática: la simulación del modelo se realiza utilizando Microsoft Excel, donde se corrieron las simulaciones para niveles variables de esfuerzo de pesca industrial (entre 0 y 22.000) y distintos tamaños de reserva del ARPA(entre 0 y $70 \%$ del área). Es importante considerar que los resultados están bajo condición de equilibrio (crecimiento de la biomasa igual a cero), por ende, todos los resultados son producto de la estática comparativa, es decir, se unen las condiciones de equilibrio que dan cuenta sobre el movimiento real de un equilibrio a otro.

La condición inicial del modelo considera la 
biomasa estimada en el año 2002, puesto que, es la última biomasa estimada y es la que estaría dando cuenta de la situación actual del recurso. Para el parámetro, tasa intrínseca de crecimiento, se ha tomado el valor de menor crecimiento posible a un $95 \%$ de confianza, dado el escaso reclutamiento que ha experimentado el recurso. Lo anterior hace pensar que la población no está creciendo según lo esperado. Por otra parte, se ha considerado el valor mayor del coeficiente de capturabilidad a un $95 \%$ de confianza, permitiendo afirmar que la flota está operando al máximo de eficiencia. Además si se considera el esfuerzo artesanal constante, se obtienen los siguientes parámetros y los valores iniciales de las variables de estado (Tabla 1).

Parámetros económicos: los precios y costos (reales) se han estimado en el periodo 1991-2001. Se considera el precio medio de tonelada de harina

Tabla 2. Valores de las variables de estado y parámetros del modelo.

Table 2. State variables values and parameters of the pattern.

\begin{tabular}{cc}
\hline Variables endógenas & Valor inicial \\
\hline $\mathrm{X}_{0}$ (ton) & 7.398 .764 \\
$\mathrm{X}_{1}$ (ton) & $\alpha \mathrm{X}_{\mathrm{F} 0}$ \\
$\mathrm{X}_{2}$ (ton) & $(1-\alpha) \mathrm{X}_{\mathrm{F} 0}$ \\
\hline Parámetro & Valor \\
\hline$\gamma_{F}$ & 0,16 \\
$X_{\max }$ (ton) & 20.600 .469 \\
$b$ (ton) & 1.894 .123 \\
$\mathrm{q}_{\mathrm{A}}$ & $9,9404 \mathrm{E}-07$ \\
$\mathrm{E}_{\mathrm{A}}($ Viajes totales $)$ & 6.066 \\
$\mathrm{q}_{\mathrm{I}}$ & $8,271 \mathrm{E}-06$ \\
$\eta$ & 0,21 \\
$\mathrm{P}_{\mathrm{H}}(\mathrm{US} \$)$ & 537 \\
$\mathrm{C}_{\mathrm{P}}(\mathrm{US} \$)$ & 145 \\
$\mathrm{C}_{\mathrm{E}}(\mathrm{US} \$)$ & 5.330 \\
$\mathrm{C}_{\mathrm{A}}(\mathrm{USS} \$)$ & 50 \\
\hline
\end{tabular}

de pescado $\left(\mathrm{P}_{\mathrm{H}}\right)$, periodo que estaría expresado en un promedio de 537 US\$ ton $^{-1}$. Para el costo asociado al proceso de elaboración $\left(\mathrm{C}_{\mathrm{p}}\right)$, se ha considerado un promedio de $145 \mathrm{US} \$ \cdot \operatorname{ton}^{-1}$, tomado de Palma et al. (2002). De este mismo trabajo y para el mismo periodo, se han tomado los siguientes costos: costo por tonelada de captura en pesca artesanal $\left(\mathrm{C}_{\mathrm{A}}\right)$ de 50 US\$ ton $^{-1}$ y costo por la unidad de esfuerzo o costo de viaje de una unidad de la flota industrial $\left(\mathrm{C}_{\mathrm{E}}\right)$ de 5.330 US\$ viaje $^{-1}$.

\section{RESULTADOS}

\section{Simulación estática}

En el área de reserva a la pesca artesanal, en la medida que el nivel de protección va en aumento, los niveles de biomasa en equilibrio también aumentan, lo que posibilita aumentos de esfuerzos de pesca industrial. Por otra parte, en situaciones de protección inferior a un 30\% (nivel promedio de protección alcanzado en el ARPA, en la zona norte, considerado desde la primera milla al año 2002), la biomasa en equilibrio en la reserva artesanal tiende a la baja en la medida que el esfuerzo de pesca industrial aumenta y en caso contrario, aumenta o tiende a permanecer constante. Esto se debe entender en el contexto de la influencia del área de pesca sobre el área de reserva artesanal, mediante la acción de la flota industrial en condiciones de equilibrio (Fig. 1).

En relación a la biomasa en equilibrio en el área de pesca, se puede apreciar dos situaciones enmarcadas antes y después de las 12.000 unidades de esfuerzo. Para valores menores, es posible ver que a mayores niveles de protección dado un esfuerzo industrial cualquiera, las biomasas equilibran a niveles menores, ya que la biomasa depende del área de protección, es decir, a mayor reserva menor biomasa en área de pesca industrial. Luego para esfuerzos superiores a 12.0000 unidades, la situación se revierte completamente; es decir, a mayores niveles de protección, dado un esfuerzo cualquiera, las biomasas equilibran a niveles mayores (Fig. 2).

No se debe olvidar que bajo condición de equilibrio la captura sustentable es igual al excedente productivo. Aumentando el tamaño de la reserva se tendrán dos efectos principales: se restringirá el área disponible para la pesca industrial, pero aumentará el excedente productivo disponible para la industrial debido a los efectos de migración de peces. Cuando el esfuerzo de pesca industrial crece, las capturas 


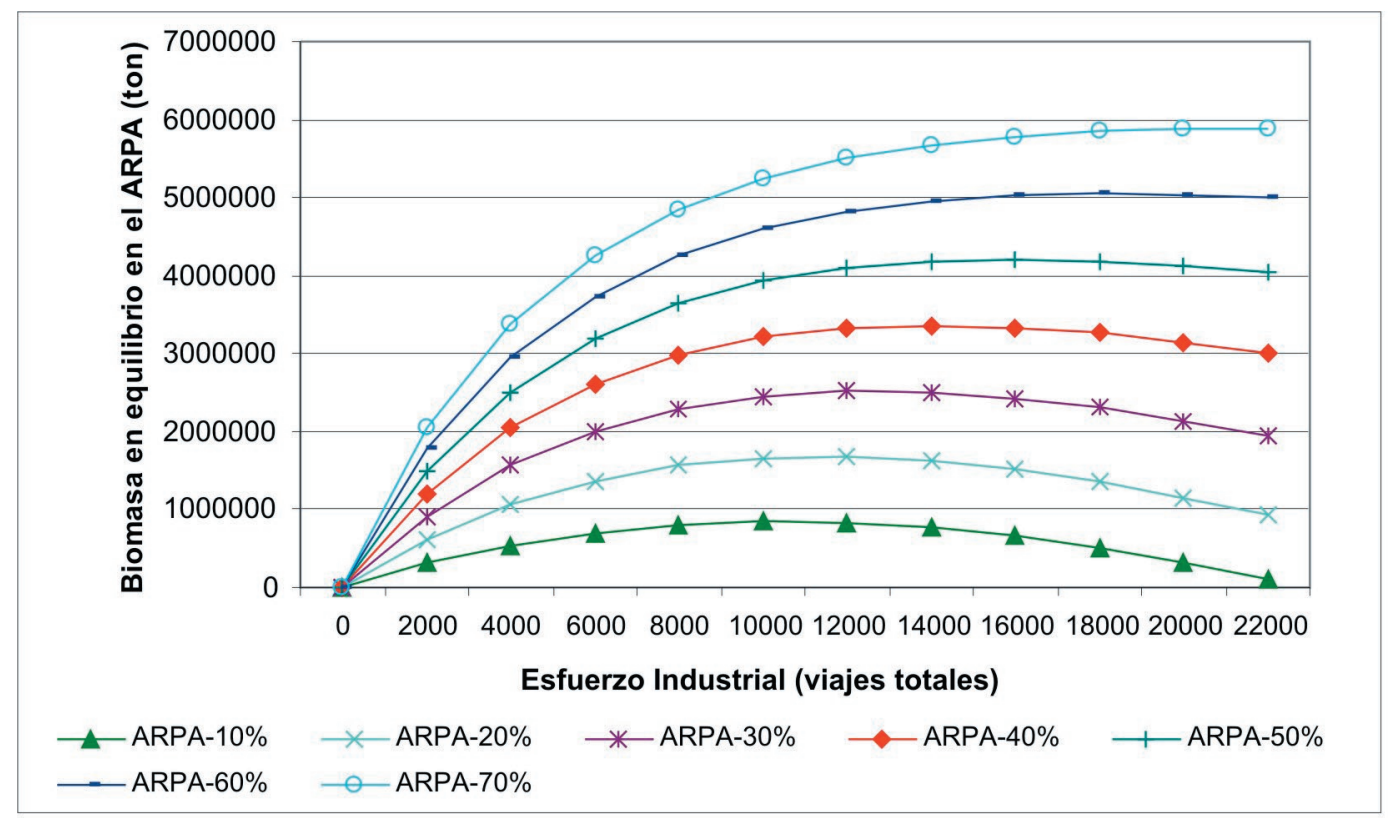

Figura 1. Evolución de la biomasa en equilibrio en función del esfuerzo, a distintos niveles de protección del área de reserva a la pesca artesanal.

Figure 1. In balance biomass evolution in function of the effort at different levels of protection of the reservation area to the artisanal fishermen.

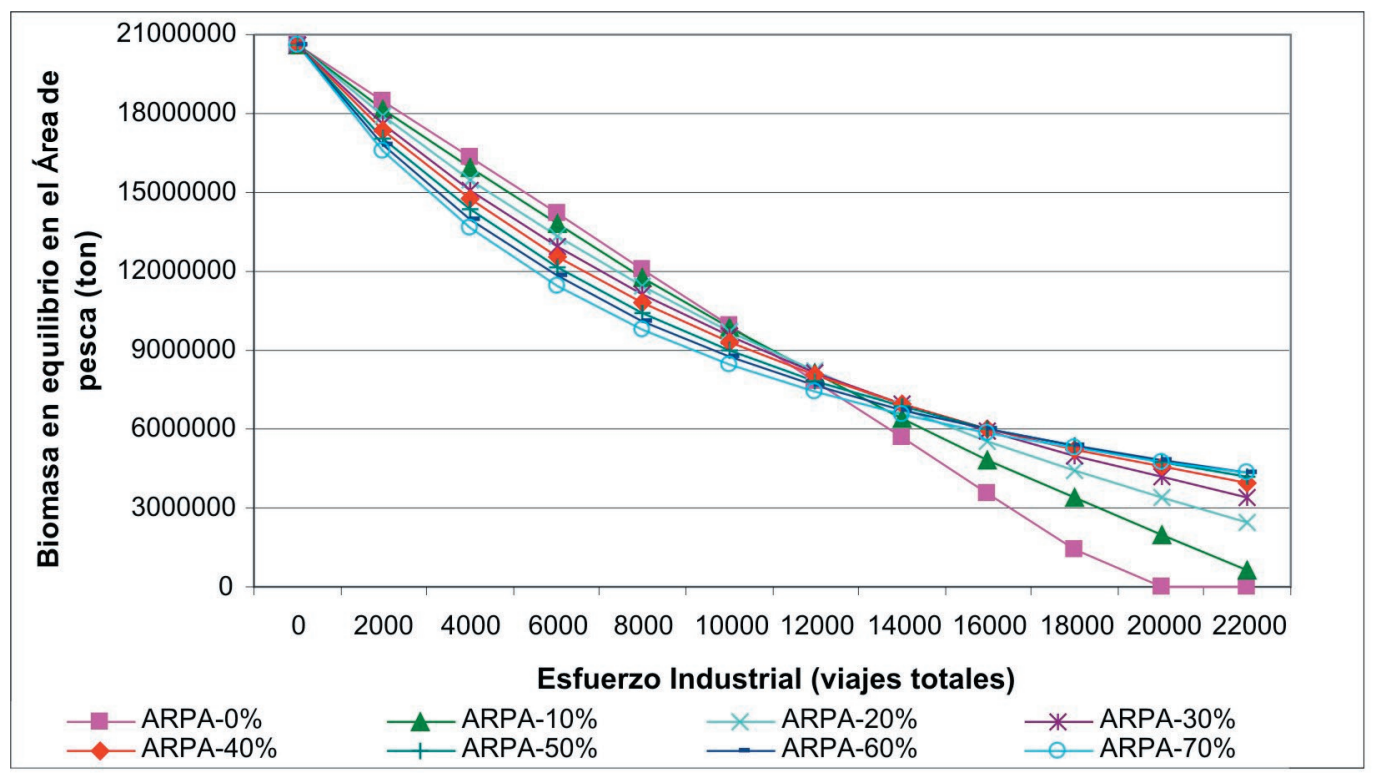

Figura 2. Evolución de la biomasa en equilibrio en función del esfuerzo, a distintos niveles de protección del área de pesca industrial.

Figure 2. In balance biomass evolution in function of the effort at different levels of protection of the reservation area to the industrial fishing. 
tienden a depender exclusivamente de la transferencia neta de biomasa de peces (Fig. 2).

Otro hecho importante relacionado con los excedentes productivos son las cuotas globales anuales de captura, las que no debieran sobrepasar los excedentes productivos. El DS MINECON No 1097 de 2002, establece una cuota global de captura para la anchoveta en el año 2003 para la zona norte de 428.625 ton. Posteriormente, se aumenta dicha cuota a 608.624 ton. Finalmente el DS N ${ }^{\circ} 237$, aumenta la cuota global anual de captura para la pesquería de la anchoveta en ambas regiones a 964.812 ton, valor desglosado en los subsectores artesanal e industrial, con 117.116 y 847.696 ton respectivamente. La cantidad asignada en el último decreto no es concordante con el valor estimado en esta investigación, ya que, a un nivel de protección del $30 \%$ y a un nivel de esfuerzo equivalente al observado el 2001 (12.000 unidades de esfuerzo), se obtiene un excedente productivo de 1.166.116 ton. Esta mayor cantidad se explica por el efecto de protección del ARPA en la estimación de los excedentes (Fig. 3).

Por otra parte, se tiene excedentes productivos crecientes en función de la protección, pero esto no genera mayor captura industrial porque esos excedentes crecen con el tamaño del área de pesca artesanal, donde el esfuerzo artesanal es constante, lo que implica excedentes sin capturar. Por tanto, no se esperan los beneficios deseados de la pesquería (Fig. 3).

Dada las condiciones actuales del esfuerzo aplicado, vale decir, 12.000 unidades y estableciendo la relación de beneficio de la pesquería (bajo condiciones de equilibrio), es posible afirmar que el equilibrio bioeconómico (nivel de esfuerzo donde los costos totales igualan a los ingresos totales), se alcanzará a 15.000 unidades del esfuerzo. Por tanto, pasado este nivel no existen incentivos para aumentar el esfuerzo en la pesquería y esto juega a favor de la protección del recurso (Fig. 4).

Nuevamente, si se toma el esfuerzo en los 12.000 viajes totales y se observa el comportamiento de los porcentajes de protección del ARPA 25\%,30\% y $45 \%$, son los que garantizan los máximos beneficios en la pesquería para ese nivel de esfuerzo, siendo sus valores US $\$ 16.100 .000$, US $\$ 15.900 .000$ y US\$15.900.000, respectivamente. Por otra parte, cuando se aumentan los esfuerzos hacia 15.000 viajes totales, valor donde se obtiene el equilibrio bioeconómico, son las protecciones de $20 \%, 30 \%$ y $40 \%$ del ARPA que mantienen los máximos beneficios de la pesquería (Fig. 4).

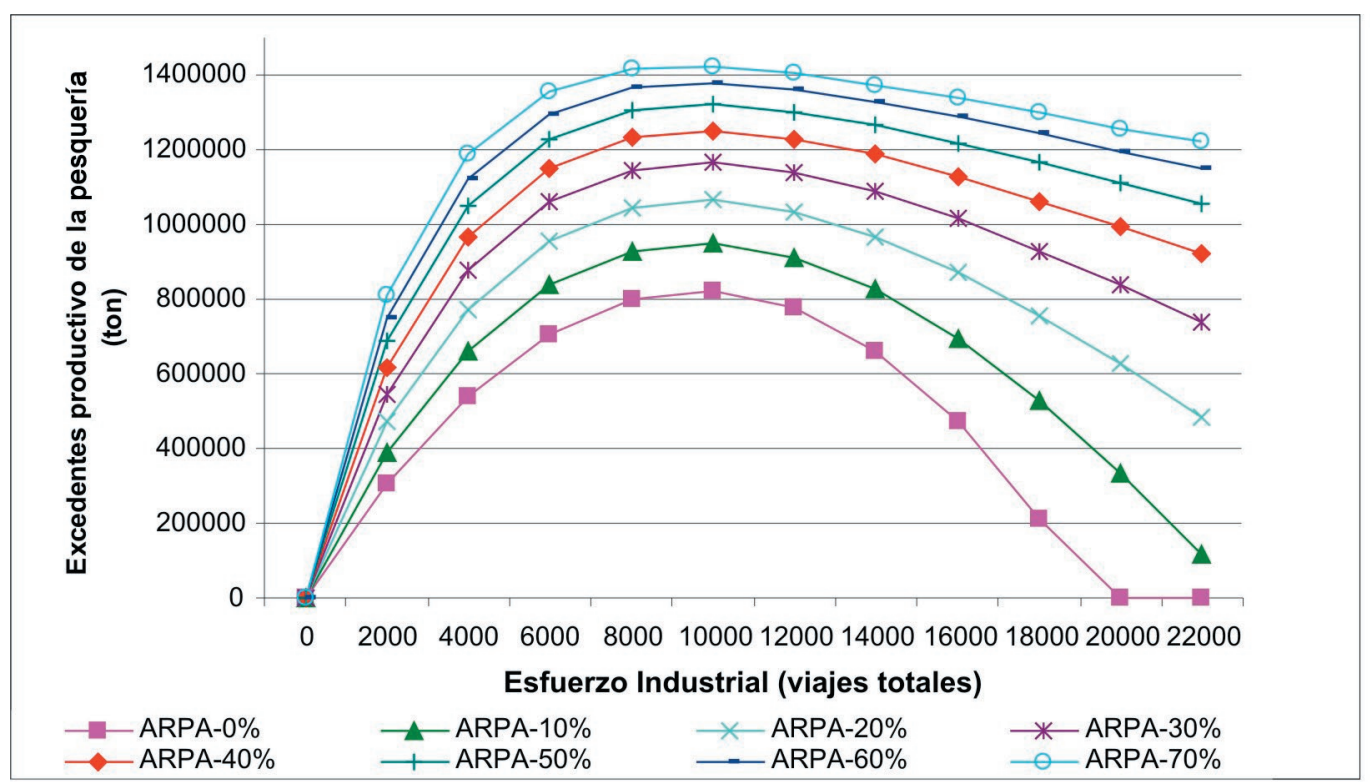

Figura 3. Evolución del excedente productivo de la pesquería en función del esfuerzo, a distintos niveles de protección del área de pesca artesanal.

Figure 3. Surplus productive evolution of the fishery in function of the effort at different levels of protection area to the artisanal fishermen. 


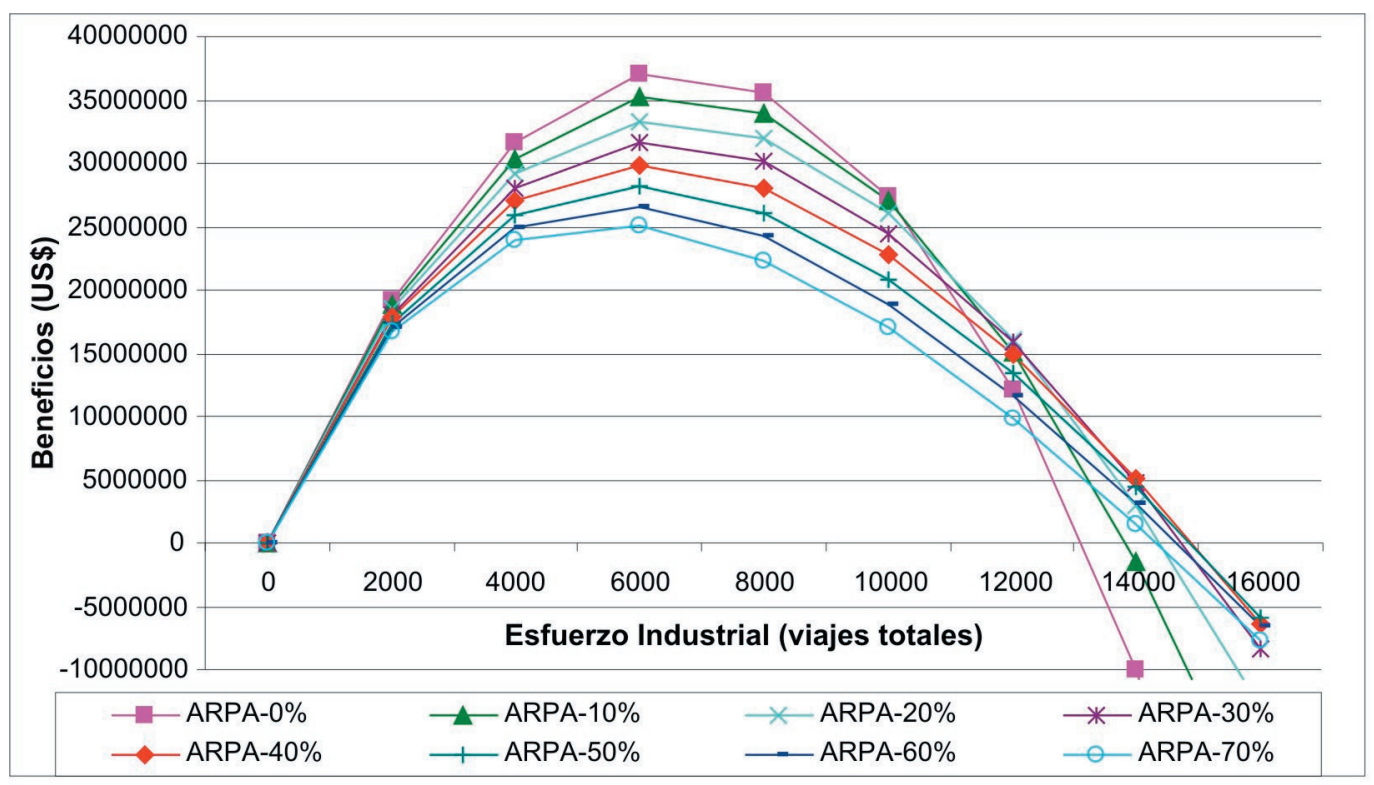

Figura 4. Beneficios de la pesquería a distintos niveles de protección.

Figure 4. Fishery benefits at different protection levels.

\section{Simulación dinámica}

Se fija una biomasa inicial de 7.398.764 ton y además se inicia la simulación con un esfuerzo de 11.000 viajes por año. En relación con el parámetro de tasa intrínseca de crecimiento, se ha tomado el menor valor posible a un $95 \%$ de confianza, dado el nivel de reclutamiento que ha experimentado la pesquería en el último año y con relación a los otros parámetros, estos son los mismos valores que se emplearon en la simulación estática.

\section{Comportamiento de la biomasa en los regímenes de regulación}

En el caso del acceso abierto (AA) las biomasas caen rápidamente para converger a valores que van desde 6.723.257 a 7.366.971 ton a diferentes niveles de protección. En la situación de Cuota Total Permisible (CTP) los valores de convergencia de las biomasas van desde 6.742.419 a 8.170.127 ton. Claramente los niveles de biomasa con AA, están por debajo de la CTP. No obstante, en el régimen CTP para niveles de reserva de $15 \%$ y $30 \%$ a la pesca artesanal, no se logra aumentos de la biomasa en la convergencia como se esperaría al generar como medida de manejo de la pesquería una cuota global. En cambio, para un nivel de protección del $45 \%$ en regulación CTP, es posible esperar aumentos en la biomasa (Fig. 5).
Es importante considerar, de acuerdo al modelamiento presentado, cualquier forma de protección del recurso para conseguir aumentos de biomasa, dadas las condiciones de la pesquería y considerando que no existen políticas de aumentos del esfuerzo de la pesca artesanal, se logrará a expensas de los beneficios posibles de obtener de la actividad industrial de la pesquería. En tal sentido, cuando se está bajo el régimen CTP con niveles de protección de $45 \%$ y $60 \%$, se obtienen beneficios negativos que convergen a los siguiente valores: US\$ -10.783.241 y US\$ $-4,629,852$, respectivamente. Sin embargo, en el régimen de $\mathrm{AA}$, es decir, cuando la industria ajusta su esfuerzo en función de sus beneficios, es posible obtener beneficios positivos que convergen a valores entre US\$500.898 y US\$7.760.031, dependiendo del nivel de protección. Por otro lado, el nivel de protección de un $15 \%$ en régimen CTP entrega un beneficio muy bajo, cercano a cero (Fig. 6).

Como era de esperar, el esfuerzo aumenta a distintos niveles de protección, en particular en AA, lo que representa un aumento de embarcaciones en la pesquería, lo que también se reflejaría en una disminución de la biomasa a todos los niveles de protección. Luego, los esfuerzos caen rápidamente, debido a que los beneficios empiezan a tomar valores negativos. Para niveles en AA con protecciones de $15 \%, 30 \%, 45 \%$ y $60 \%$, los esfuerzos convergen 


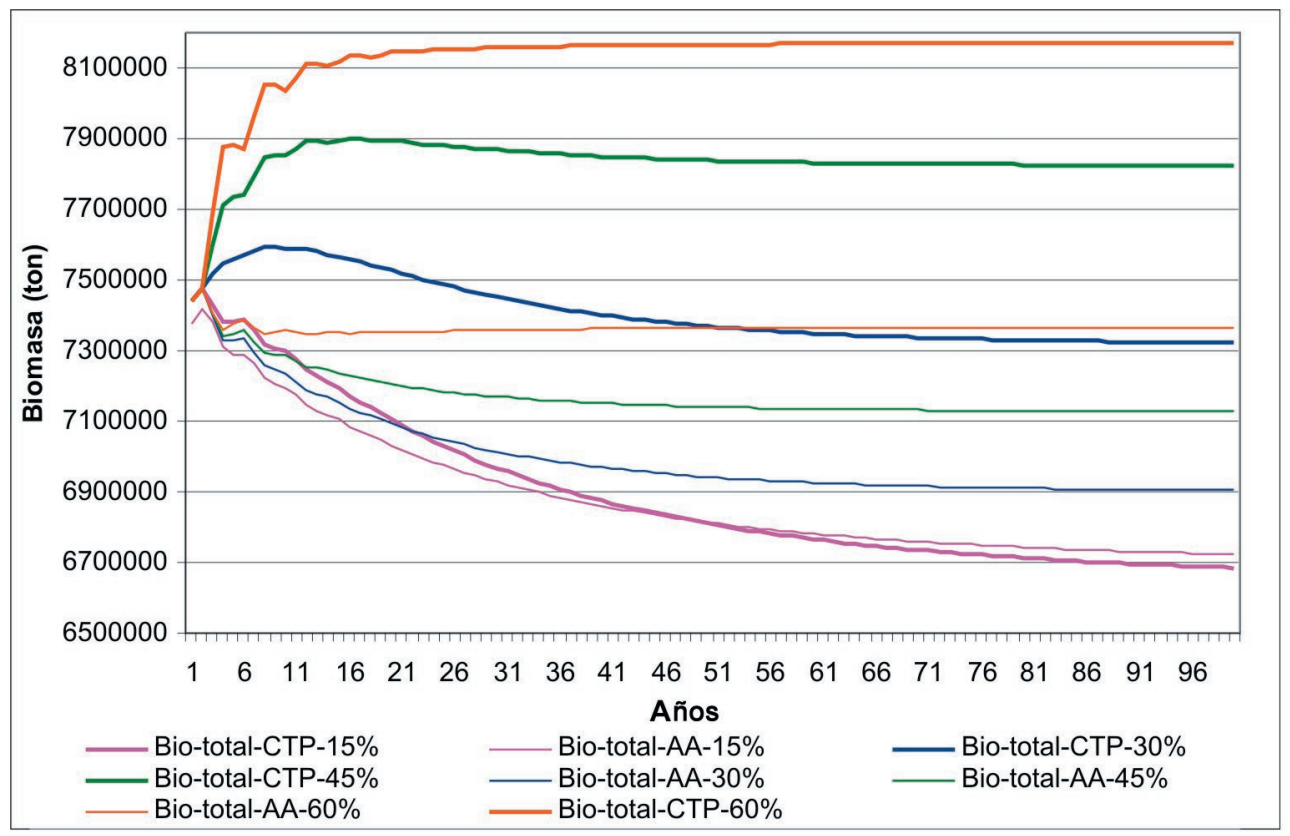

Figura 5. Evolución de la biomasa de la pesquería a distintos niveles de protección, bajo dos regímenes de regulación AA y CTP.

Figure 5. Fishery biomass evolution at different protection levels under two administrations AA and CTP.

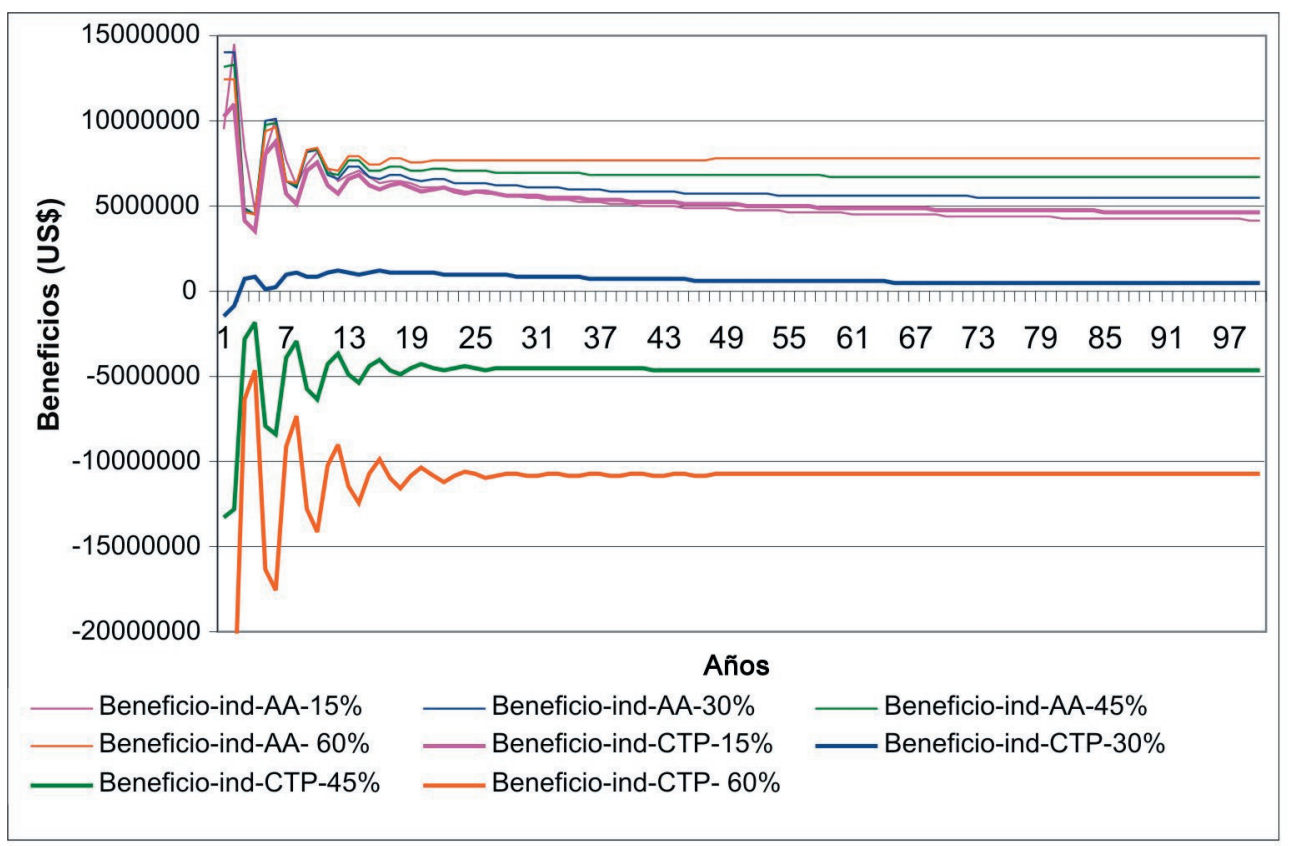

Figura 6. Evolución del beneficio industrial de la pesquería a distintos niveles de protección, bajo dos regímenes de regulación AA y CTP.

Figure 6. Industrial benefit evolution of the fishery at different protection levels, under two administrations AA and CTP. 
a: $11.507,11.557,11.808$ y 11.931 viajes totales respectivamente. Además, estos valores no difieren demasiado entre niveles de protección del ARPA (Fig. 7).

En la situación de AA, dependiendo del nivel de protección los esfuerzos convergen a valores que están de 12.000 a 12.700 viajes totales respectivamente. En el caso de CTP el nivel que presenta un comportamiento similar a los de AA, corresponde al nivel de protección de un 30\%, convergiendo a 12.000 unidades de esfuerzo. Finalmente, en CTP, en los otros casos de reserva, las convergencias van desde $9.706,10.447$ y 11.664 viajes totales para niveles de protección de $60 \%, 45 \%$ y $30 \%$, respectivamente (Fig. 7).

\section{DISCUSIÓN}

El modelamiento realizado ha permitido analizar la pesquería como monoespecífica. El parámetro más sensible ha sido la tasa intrínseca de crecimiento, lo que evidencia la necesidad de tener precaución a la hora de interpretar los resultados, ya que este parámetro de alguna forma da cuenta del estado del recurso en términos de desove, reclutamiento y nivel del stock.
Aliaga et al. (2001), según su modelamiento manifiestan que la flota está ejerciendo esfuerzos superiores al del punto de equilibrio de libre AA y reafirman lo anterior con la hipótesis que la flota ejerce esfuerzos superiores al de equilibrio en AA, cuando existe integración vertical, dado que mediante el proceso, la industria recupera la pérdida de la flota. También, Cerda et al. (1997) señalan que es importante notar que existe un cierto grado de integración vertical. Al respecto y de acuerdo a los resultados presentados, se puede afirmar que los esfuerzos están por debajo del punto de equilibrio en AA.

A pesar de todas las limitaciones del modelamiento en la parte estática, es decir, las uniones de varias situaciones en equilibrio, las cuales es importante recordar que no dan la información sobre el movimiento real de un equilibrio a otro, se ha podido tener una visión bastante real del comportamiento de las variables modeladas, en especial en relación con el esfuerzo pesquero, el cual se ha estabilizado por debajo del equilibrio bioeconómico. Paralelamente, la simulación dinámica ha permitido observar que la senda del esfuerzo converge al valor del 2002 de la pesquería. Esto no es fácil de explicar, ya que la pesquería virtualmente opera en AA y en este contexto la literatura señala que ocurriría el fenómeno llamado "carrera olímpica", como lo señalan Conrad \& Clark

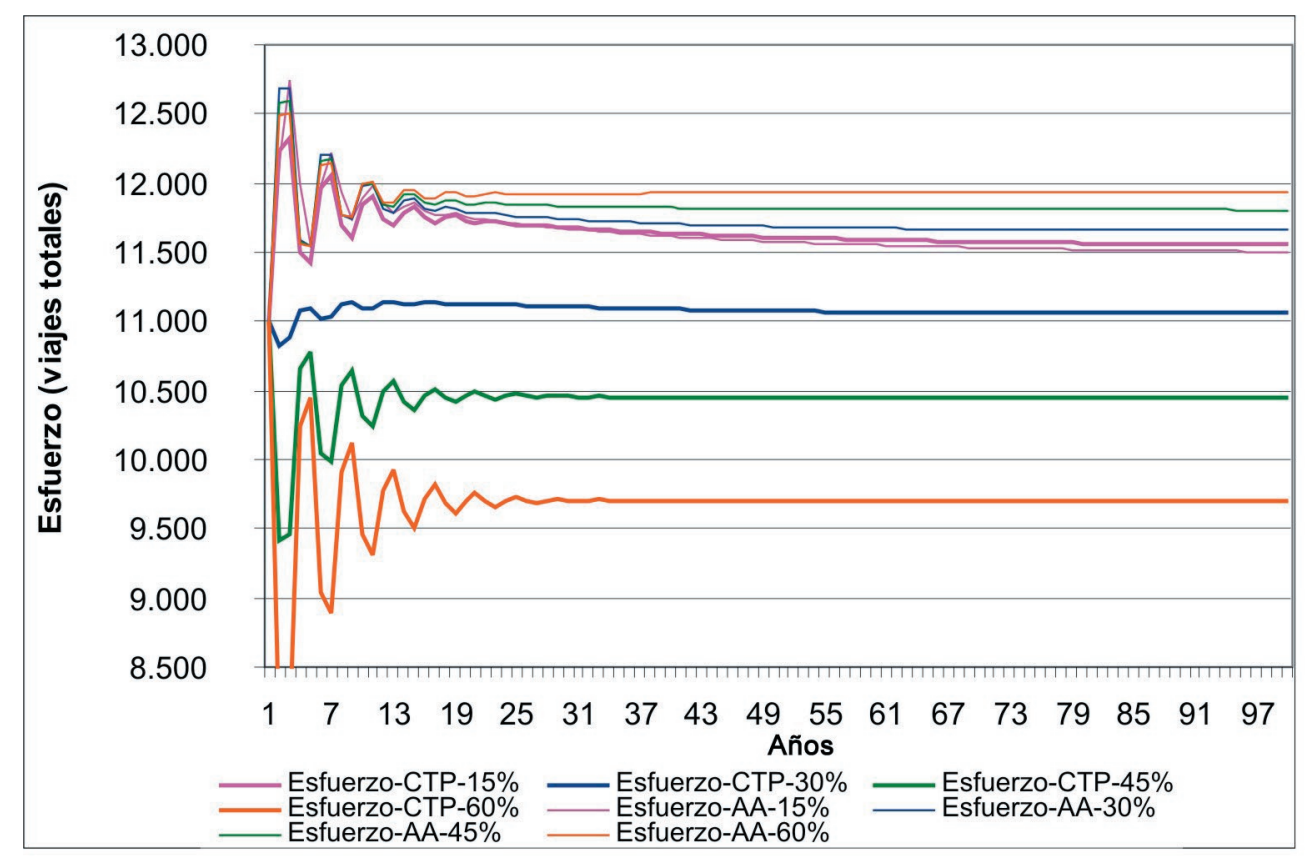

Figura 7. Evolución del esfuerzo a distintos niveles de protección, bajo los regímenes de regulación AA y CTP.

Figure 7. Effort evolution at different protection levels under the administrations AA and CTP. 
(1987), Clark (1996) y Anderson (2000). Lo anterior inevitablemente llevaría a una sobreinversión en medios de pesca más eficientes para enfrentar de mejor manera la competencia por el acceso al recurso en un escenario de rendimientos decrecientes, lo que se traduce en un aumento del valor de la tonelada desembarcada, producto de la disminución de la captura. Es importante destacar que esta situación ha sido superada y complementada con la aplicación de la medida de administración Límite Máximo de Captura por Armador (LMCA).

Desde el punto de vista de modelamiento, este trabajo ha considerado el problema de la verticalidad, incorporando el valor del precio de la harina en la función del ingreso, de la misma manera que Salgado (2000) en la pesquería del jurel. Esta situación es más real ya que existe un mercado para harina, por ende un precio, con esto se soluciona el problema de falta de mercado para la captura. Esto permite tener una visión global del problema y es posible entender mejor cómo los agentes se han ajustado a los cambios del sistema. Por otra parte, los resultados generados y contrastados con la realidad, más específicamente lo que dice relación con el esfuerzo de pesca, permiten afirmar que este se ubica por debajo del equilibrio bioeconómico y se estabiliza, dadas las condiciones actuales del recurso, muy cercano al valor que la pesquería presenta en la actualidad.

Una posible explicación a la problemática planteada, en términos globales, puede ser entendida por el ajuste operacional y tecnológico realizado por los agentes pesqueros, vale decir, sustitución de naves y fusiones de empresas. Por otra parte, el restringir la operación de la flota en el ARPA, se genera el desarrollo de ventanas de penetración a contar del 1994. Además se agrega el monitoreo, control y vigilancia de la actividad pesquera, mediante el posicionamiento satelital, a contar de agosto del 2000 y la medida de administración LMCA. Todo esto, en su conjunto podría explicar que el esfuerzo pesquero esté bajo el punto de equilibrio bioeconómico y no esté en presencia de sobreinversión.

Los resultados del modelamiento estático y dinámico, permiten entender en alguna medida el rol que juega el proteger una parte del stock del recurso, en este caso en particular proteger una fracción del ARPA, puesto que permite regular el esfuerzo en forma indirecta por medio de la fracción protegida y de esta manera obligar al agente pesquero a ubicarse antes del equilibrio bioeconómico. Desde este punto de vista, la protección del ARPA puede constituir una herramienta eficiente en la administración de la pesquería. Además, es importante destacar que la protección de una fracción del ARPA, ha generado beneficios en la pesquería en el sentido de tener biomasas libres del esfuerzo pesquero. De ahí la necesidad de generar áreas de reservas, ya que las reserva significan tener stock de peces más resilentes a la explotación, con lo cual se reduce el riesgo del colapso del stock y de las pesquerías. Son los proveedores de biomasa que se transfieren al área de pesca. Las reservas además, se pueden considerar como un medio de conducta relacionada con las incertidumbres de la valoración del stock y el control del esfuerzo de pesca en la administración de las pesquerías, tal como lo han propuesto Boncoeur et al. (2002), Guénette et al. (1998) y Lauck et al. (1998).

Desde el punto vista de los beneficios, en la simulación estática cuando se está sobre las 12.000 unidades de esfuerzo, los beneficios empiezan a bajar rápidamente en los niveles de protección muy altos $(60 \%$ y $70 \%)$ y muy bajos, $(0 \%, 10 \%$ y $20 \%)$. Esto se explica porque la captura empieza a depender en gran medida de la transferencia de biomasa que se origina en la reserva hacia el área de pesca. En cambio, en simulación dinámica, en la situación de AA no existen grandes diferencias en los beneficios entre los niveles de reserva, la senda de convergencia es similar y está en torno a valores no muy diferentes. En contraposición con la CTP, cuando los resultados de los beneficios en la convergencia son muy dispares entre niveles de protección, donde los beneficios son inversamente proporcionales respecto al nivel de protección, con esto es posible captar el corolario propuesto por Boncoeur et al. (2002), en que el tamaño óptimo de la reserva (protección del ARPA), según la dirección global del análisis costo beneficio, es más grande que el óptimo si es el único objetivo de la administración de la pesquería, ya que existe pérdida marginal neta por la industria pesquera, inducida por un aumento en el tamaño relativo de la reserva que en términos absolutos corresponde a una ganancia marginal que se queda en la reserva, a diferencia a lo planteado por Boncoeur et al. (2002), donde la ganancia marginal es traspasada a otro agente del sistema, es decir, al sector dedicado al ecoturismo. En la situación de la pesquería de la anchoveta debiera ser capturada por el sector artesanal, cosa que no es factible dado que este sector no puede crecer.

Uno de los principales aspectos críticos del modelamiento, dice relación con la tasa intrínseca de crecimiento $\left(\gamma_{F}\right) y$ el coeficiente de transferencia 
de biomasa (b) como lo presenta Anderson (2000), pero para una situación de transferencia de biomasas dependientes de las densidades. No obstante, en esta situación mediante el análisis de sensibilidad de los parámetros, es posible inferir que el parámetro que más influye en los resultados es el parámetro $\gamma_{F}$. Por otra parte, el coeficiente de transferencia de biomasa, a pesar de no impactar mayormente en los resultados, interviene en que el gran supuesto de excedentes productivos es igual a la captura, en la medida que el esfuerzo crece, va a depender principalmente de la transferencia de biomasa de la reserva hacia la zona de pesca. Al respecto, este problema debiera ser respaldado por evidencia empírica, puesto que en esta investigación se ha considerado el modelamiento teórico de la transferencia propuesto por Sanchirico \& Wilen (2001).

Al igual como lo señalan Boncoeur et al. (2002), el ARPA sin ser una reserva propiamente tal, ha generado los mismos conflictos que si lo fuera, es decir, conflictos entre los agentes económicos involucrados. Dan cuenta de estos los informes técnicos desarrollados por el consejo zonal de pesca. Por otra parte, este tratamiento o modelamiento del problema ha permitido evaluar claramente los beneficios y ha permitido conducir variaciones en la experiencia, aportando más de lo que se pudiera hacer empíricamente, como lo afirman diversos autores (Bohnsack, 1996; Parrish, 1999; Jennings, 2001; Li, 2001; Mangel, 2001). Por otra parte, la discusión económica de las reservas marinas se ha enfocado principalmente en su uso como una herramienta de dirección de pesquerías. Es así que Holland \& Brazee (1996) han demostrado que las reservas marinas podrían mejorar las capturas sustentables en las pesquerías sobreexplotadas, dado un nivel fijo de esfuerzo de pesca.

Finalmente, Hannesson (1998) y Anderson (2000), han cuestionado la utilidad de las reservas marinas como una herramienta para la dirección de las pesquerías en un contexto determinístico con acceso abierto. Al respecto, los resultados presentados en el presente trabajo demuestran que en condiciones de AA y con muy baja tasa intrínseca de crecimiento de la población a distintos niveles de protección, entrega mejores beneficios para el sector industrial.

\section{CONCLUSIONES}

De acuerdo al modelamiento planteado y a la luz de sus resultados obtenidos, es posible manejar la pesquería, mediante la determinación del porcentaje del
ARPA que se otorga al sector industrial vía ventana de penetración, y se es capaz de garantizar la captura de los pescadores artesanales.

De acuerdo a las condiciones del recurso al 2002 y considerando el modelamiento estático, en la medida que el esfuerzo industrial aumenta y se acerca al punto de equilibrio bioeconómico, los niveles de protección en torno al 30\% (nivel de protección del ARPA al 2002, medido desde la primera milla), garantizan los máximos beneficios de la pesquería. Además, el equilibrio bioeconómico se obtiene con un nivel de esfuerzo de 15.000 viajes totales.

En el modelamiento dinámico, considerando las mismas condiciones del recurso que en el modelamiento estático, es posible ver que la política de CTP logra aumentar la biomasa en todos los niveles de protección sobre la política de AA, pero se observan beneficios negativos y en el caso positivo, inferior a AA.

\section{REFERENCIAS}

Agüero, M. \& E. González. 1996. Managing transboundary stocks of small pelagic fish. Problems and options. World Bank Discussion. Paper No329 (Fisheries Series). The World Bank, Washington DC, 40 pp.

Aliaga, B., D. Gómez \& S. Neira. 2001. Análisis bioeconómico de la pesquería de sardina (Sardinops sagax) y anchoveta (Engraulis ringens) de la zona norte de Chile. Invest. Mar., Valparaíso, 29(2): 15-23.

Anderson, L. 2000. The effects of ITQ implementation: a dynamic approach. Nat. Resour. Model., 13(4): 435-470.

Bohnsack, J.A. 1996. Marine reserves, zoning and future of fishery management. Fisheries, 21(9): 14-16.

Boncoeur, J., F.Alban, O. Guyader \& O. Thébaud. 2002. Fish, fishers, seals and tourists: economic consequences of creating a marine reserve in a multi-species, multi-activity context. Nat. Resour. Model., 15(4): 387-411.

Böhm M.G. \& M.A. Barbieri. 1996. Análisis de la captura y el esfuerzo de pesca de la unidad de pesquería de sardina española y anchoveta de la zona norte. Proyecto FIP 93-21, 139 pp.

Cerda, A., B. Aliaga, E. Bobenrieth, J. Dresdner, R. Quiñones, A. Sepúlveda, M. Gálvez \& A. 
Hernández. 1997. Evaluación bioeconómica de la pesquería industrial del jurel en la zona Centro Sur. Proyecto FIP No 95-12, 228 pp.

Claramunt, G.,M.Araya \& R.Roa.2003. Dinámica de poblaciones explotadas. Universidad Arturo Prat. Proyecto Modernización de la Enseñanza de las Ciencias Biológicas Básicas (MECESUP $\mathrm{UAP} / 001), 144 \mathrm{pp}$.

Clark, C.W. 1996. Marine reserves and the precautionary management of fisheries. Ecol. Appl., 6(2): 369-370.

Conrad, J.M. \& C.W. Clark. 1987. Natural resource economics. Notes and problems. Cambridge University Press, London, 229 pp.

Díaz, J.P., M. Aguilar \& X. Cancino. 1997. Evaluación económica de las embarcaciones de pequeños armadores, al operar en los caladeros de embarcaciones industriales, en el norte de Chile. VII Congreso Latinoamericano de Ciencias del Mar. Instituto Oceanográfico de la Universidad de Santos, Brazil.

Gordon, H.S. 1954. The economics of a common property resource: the fishery. J. Polit. Econ., 62: 124-142.

Guénette, S., T. Lauck \& C.W. Clark. 1998. Marine reserves: from Beverton and Holt to the present. Rev. Fish. Biol. Fisheries, 8: 251-272.

Hannesson, R. 1998. Marine reserves: what would they accomplish? Mar. Resour. Econ., 13: 159170.

Holland, D.S. \& R.J. Brazee. 1996. Marine reserves for fisheries management. Mar. Resour. Econ., 11: 157-171.

Instituto de Fomento Pesquero (IFOP). 2003. Investigación y evaluación del stock de anchoveta y sardina en las I y II Regiones. Informe final. Valparaíso, $39 \mathrm{pp}$.

Jennings, S. 2001. Patterns and prediction of population recovery in marine reserves. Rev. Fish. Biol. Fisheries, 10(4): 209-231.

Lauck, T., C.W. Clark, M. Mangel \& G.R. Munro. 1998. Implementing the precautionary principle in fisheries management through marine reserves. Ecol. Appl., 8 (Suppl. 1): 572578.

Li, E. 2001. Optimum harvesting with marine reserves. North Amer. J. Fish. Manage., 20(4): 882-896.

Mangel, M. 2001. Irreducible uncertainties, sustainable fisheries and marine reserves. Evol. Ecol. Res., 2(4): 547-57.

Palma, W., M. Aguilar, J.P. Díaz \& E. Oliva. 2002. Interacción de la actividad pesquera industrial y artesanal, en la zona de reserva a la pesca artesanal en la I y II Región. Doc. Tec. Universidad Arturo Prat, Departamento Ciencias del Mar, 1- 2002: 53 pp.

Peña-Torres, J., M. Basch \& S. Vergara. 2003. Eficiencia técnica y escalas de operación en pesca pelágica: un análisis de fronteras estocásticas, Cuadernos de Economía, 40(119): 43-83.

Parrish, R. 1999. Marine reserves for fisheries management: why not. Rep. CalCOFI, Cooperative Oceanic Fisheries Investigations, 40: 77-86.

Salgado, H. 2000. Simulación de los efectos de cuotas individuales transferibles sobre la pesquería del jurel en Chile. Tesis de Magíster en Economía de Recursos Naturales y del Medio Ambiente. Universidad de Concepción, Concepción, 117 pp.

Sanchirico, J. \& J. Wilen. 2001. A bioeconomic model of marine reserve creation. Environ. Econ. Manage., 42: 257-276.

Schaefer, M.B. 1954. Some aspects of the dynamics of populations important to the management of comercial marine fisheries. Bull. Inter-Amer. Tropical Tuna Comm., 1: 25-56.

Smith, V.L. 1968. Economics of production from natural resources. Amer. Econ. Rev., 58: 409431.

Yáñez, E., A. González \& M.A. Barbieri. 1995. Estructura térmica superficial del mar, asociada a la distribución espacio-temporal de sardina y anchoveta en la zona norte de Chile entre 1987 y 1992. Invest. Mar., Valparaíso, 23: 123-147. 